\title{
Bivalence modale dans les complétives régies par des verbes épistémiques : perspective contrastive (italien/français)
}

\section{Luciana T. Soliman}

\section{CpenEdition}

\section{Journals}

Édition électronique

URL : https://journals.openedition.org/ml/229

DOI : $10.4000 / \mathrm{ml} .229$

ISSN : 2274-0511

Éditeur

Association Modèles linguistiques

Édition imprimée

Date de publication : 1 juillet 2010

Référence électronique

Luciana T. Soliman, «Bivalence modale dans les complétives régies par des verbes épistémiques perspective contrastive (italien/français) », Modèles linguistiques [En ligne], 62 | 2010, mis en ligne le 21 janvier 2013, consulté le 01 juillet 2021. URL : http://journals.openedition.org/ml/229 ; DOI : https:// doi.org/10.4000/ml.229 


\section{Bivalence modale dans les complétives régies par des verbes épistémiques : perspective contrastive (italien/français) ${ }^{1}$}

\section{Luciana T. Soliman}

\section{Entrée en matière}

Si le subjonctif (SUBJ) en français contemporain a fait couler beaucoup d'encre, l'intérêt que l'écart de la norme suscite - ce que nous appelons le non-subjonctif (NON-SUBJ) - a été traité plus marginalement, la question $\mathrm{du}$ choix orientant de la part de l'énonciateur, en particulier dans l'interrogation, étant probablement l'élément le plus saillant. En italien contemporain, où le sujet mérite d'être documenté par le menu en raison de sa portée, notamment dans le domaine des verbes épistémiques gouvernant soit l'indicativo (INDi) soit le congiuntivo (CONG [fr. subjonctif, ndlr]), le choix serait principalement d'ordre stylistique. Certaines remarques de type traductologique italien/français confortent cette option.

C'est à l'occasion d'un important colloque « Intorno al congiuntivo » (Forlì, 2000) qu'a été soulevé le problème spécifique de la surprenante alternance entre l'IND $\mathrm{D}_{\mathrm{i}}$ et le CONG après le verbe épistémique credere (Schena, Prandi, Mazzoleni 2002, p. 10). Cette alternance avait déjà été signalée par Bronzi (1977, p. 446) quelques années auparavant :

[a]nche se in dipendenza da [verbi] di " giudizio problematico » una competenza di tipo toscano sente ancora come più spontaneo e corretto l'uso del congiuntivo che l'uso dell'indicativo, non bisogna dimenticare che è proprio in questo tipo di contesto che l'oscillazione e l'incertezza nell'uso dei due modi si manifesta con particolare intensità.
[...] même dans la dépendance de verbes de " jugement problématique " [comme "croire", ndlr] les locuteurs de parlers de type toscan continuent de sentir spontanément l'emploi du subjonctif comme plus correct que celui de l'indicatif ; il ne faut pas oublier que c'est dans ce genre de contexte que les fluctuations entre les deux modes sont le plus marquées.

1. Sauf à partir de l'exemple [41], les citations en italien ont été traduites par Alessandro Leiduan, maître de conférences à l'Université du Sud Toulon-Var. Qu'il soit vivement remercié. 
De leur côté, Lepschy et Lepschy (1977, p. 79) signalent brièvement :

[...] a more and more limited use of the subjunctive, replaced in subordinate clauses by the indicative, in conformity with informal spoken style, as in credo che parte «I think he is leaving», spero che sta bene «I hope he is well». This is avoided in formal literary Italian.
[...] l'usage de plus en plus réduit du subjonctif, remplacé par l'indicatif dans les propositions subordonnées, conformément à la langue familière, par exemple dans credo che parte " je pense qu'il est sur le départ », spero che sta bene "J'espère qu'il va bien ». On évite cet emploi dans la langue soutenue.

Convenons-en, si le CONG s'est toujours opposé à l' $\mathrm{IND}_{\mathrm{i}}$, tout comme la non-réalité, le doute ou bien la prise de distance se sont opposés à la réalité et à l'adhésion de l'énonciateur, aujourd'hui sa portée sémantique est devenue moins importante : le CONG connaît une véritable crise. Les enseignants, qui tentent de s'affranchir de la langue parlée quotidienne, s'en sont aperçus. La Scuola media Padre Costanzo Beschi di Castiglione delle Stiviere (Mantova) en est arrivée à instituer un comité «Salviamo il congiuntivo » (2006) pour sauvegarder l'avenir de ce mode ${ }^{2}$. Le journaliste Severgnini (2007, p. 149), moins sensible aux craintes des enseignants, a déclaré que le CONG n'est nullement mort, préférant le débat au combat :

[...] il congiuntivo è morto, dicono. Omicidio, suicidio o evento accidentale? Nessuna di queste cose. Credo che si tratti della conseguenza logica di un fenomeno illogico. Sempre meno italiani, quando parlano, esprimono un dubbio; quasi tutti hanno opinioni categoriche su ogni argomento (vino e viaggi, case e calcio, sesso e sentimenti).
[...] le subjonctif est mort, dit-on. Homicide, suicide, accident ? Rien de tout cela. Je crois qu'il s'agit de la conséquence logique d'un phénomène illogique. De moins en moins d'Italiens expriment le doute en parlant. Ils ont des avis catégoriques sur tous les sujets (le vin et les voyages, la maison et le foot, le sexe et les sentiments).

À l'en croire, les Italiens trancheraient péremptoirement. Avec des positions nettes sur n'importe quel sujet, ils perdent le goût de la mesure. Ce qui reflète probablement l'attitude d'une nouvelle société qui a fait sienne l'image d'un modèle médiatique rassurant. On rejetterait le CONG, qui comporte en demi-teinte l'hésitation.

La perspective de bons observateurs du langage est moins nuancée que celle des « techniciens » :

2. http:// salviamoilcongiuntivo.blogspot.com (dernière interrogation : octobre 2007). Ce blog réunit les paladins de la consecutio temporum qui livrent bataille contre ceux qui nient la syntaxe. 
[d]opo alcuni verbi, nomi e aggettivi elencati (in particolare quelli del primo gruppo, che esprimono un'opinione o una convinzione personale), nell'italiano colloquiale non è raro incontrare l'indicativo al posto del congiuntivo, soprattutto se il soggetto della frase introdotta da che è la seconda persona singolare tu. [...] (Patota 2006, p. 124)
[...] dans la langue familière, après certains verbes (en particulier ceux du premier groupe, qui expriment une opinion ou une conviction personnelle), il n'est pas rare de trouver l'indicatif au lieu du subjonctif, surtout si le sujet de la phrase introduite par che est à la seconde personne du singulier, $t u$.

Il est conseillé d'en endiguer l'emploi en obligeant le locuteur à la vigilance :

[i]n casi del genere, l'indicativo al posto del congiuntivo può essere accettato nell'italiano parlato, ma va evitato nellitaliano scritto e anche nell'italiano parlato di tono formale. (ibidem)
[...] en l'occurrence, l'indicatif en lieu et place du subjonctif, peut-être admis dans la langue orale, mais on l'évite dans la langue écrite, et même dans la langue parlée soutenue.

Le bon usage passe notoirement au travers des interdits; ainsi, Dardano et Trifone ne manquent pas de faire des remarques qui, bien que souples, sont d'ordre prescriptif :

[n]ella lingua parlata e, soprattutto negli ultimi tempi, anche nella lingua scritta, l'uso dell'indicativo si è esteso a spese del congiuntivo: non so se tu hai capito (interrogativa indiretta) appare a molti del tutto accettabile, in luogo di non so se tu abbia capito; meno corretto appare penso che è meglio (oggettiva) in luogo di penso che sia meglio.(Dardano - Trifone 1995, p. 363)
[...] dans la langue parlée, surtout ces derniers temps, et même dans la langue écrite, l'usage de l'indicatif s'est étendu aux dépens du subjonctif : non so se tu hai capito ("je ne sais si tu as compris") : interrogation indirecte) est tout à fait acceptable pour beaucoup d'italianophones, au lieu de non so se tu abbia capito [litt. je ne sais si tu aies compris] ; penso che è meglio ("je pense que c'est mieux] : énoncé objectif) semble en revanche moins correct que penso che sia meglio [je pense que ce soit mieux].

Pourtant l'arrière-pensée de Sensini (1997, p. 267) n'est pas très différente de l'idée de ceux qui crient au danger ; il annonce un lent et impitoyable déclin dans des énoncés où l'IND $\mathrm{i}_{\mathrm{i}}$ jure affreusement avec les verbes sembrare et credere : 
[n]ell'italiano contemporaneo il congiuntivo è in crisi: incalzato dall'indicativo, che mira a usurparne il posto, esso è in lenta ma inesorabile decadenza. Il parlante, infatti, sempre più spesso dice: «Non so quanti chili di ciliegie hanno comprato» anziché «Non so quanti chili di ciliegie abbiano comprato». E fin qui niente di grave, giacché ormai nelle interrogative dirette l'indicativo, che insiste sulla realtà dell'azione, è usato sempre più spesso del congiuntivo, che invece sottolinea l'incertezza implicita nel dubbio. Più grave, invece, è il caso in cui l'indicativo sostituisce il congiuntivo in frasi come «Mi sembra che Maria è già partita» o «Credo che Paolo è tornato». In queste frasi, infatti, l'indicativo, che è il tempo della certezza e della sicurezza, fa letteralmente a pugni con i verbi reggenti mi sembra e credo che, invece, esprimono opinione, incertezza e dubbio e che, quindi, vorrebbero il congiuntivo. Insomma: altro è dire « So che hai detto la verità » e altro è dire « Credo che tu abbia detto la verità ».
[...] en italien contemporain, le subjonctif est dans une phase critique ; talonné par l'indicatif, qui tend à le supplanter, il est en pleine décadence, lentement mais inexorablement. On entend de plus en plus fréquemment: "Non so quanti chili di ciliegie hanno comprato" ["je ne sais pas combien de kilos de cerises ils ont acheté"] au lieu de "Non so quanti chili di ciliegie abbiano comprato » [litt. "je ne sais combien de kilos de cerises ils *aient acheté]. Rien de bien grave en définitive, puisque désormais dans les phrases interrogatives directes, l'indicatif, qui met l'accent sur la réalité de l'événement, est utilisé de plus en plus fréquemment à la place du subjonctif, lequel souligne l'incertitude qu'implique le doute. En revanche, plus grave est le cas où l'indicatif remplace le subjonctif dans des énoncés comme "Mi sembra che Maria è già partita " ["Il me semble que Marie est déjà partie, au lieu de sia litt. "Il me semble que Marie soit déjà partie"] ou "Credo che Paolo è tornato " ["Je crois que Paul est revenu" au lieu de litt. "Je crois que Paul soit revenu"]. En fait, dans ces énoncés, l'indicatif, qui est le mode de la certitude et de l'assurance, entre littéralement en conflit avec des verbes recteurs comme mi sembra che ["il me semble que"] et credo che ["je crois que"] qui, en revanche, expriment l'opinion, l'incertitude, le doute et qui, par voie de conséquence, devraient appeler le subjonctif. En résumé, une chose est de dire "So che hai detto la verità " ["Je sais que tu as dit la vérité"], autre chose "So che abbia detto la verità » ["Je crois que tu *aies dit la vérité"].

Envahi par le pessimisme, il craint la disparition du CONG, l'usage prenant le dessus sur la norme (p. 268) : «[a] ogni modo, quale che ne sia la causa, la crisi del congiuntivo è un dato di fatto e, purtroppo, non serviranno certo le leggi della grammatica a salvare questo modo del verbo dal pericolo di scomparire».

Les causes seraient à chercher ; i) dans le processus d'économie linguistique : le recul du CONG va de pair avec d'autres phénomènes de simplification, à savoir le progrès du che polyvalent, l'emploi de l'adjectif à fonction adverbiale, l'usage de la coordination au détriment de la subordination (Dardano-Trifone 1995, p. 364) ; ii) dans des facteurs diatopiques, le congiuntivo étant peu employé au Centre et au Sud (cf. ibidem). 
Cette dérive modale est généralement imputable à une simplification morphosyntaxique. Peut-être, De Mauro (1970, p. 192, apud Bronzi 1977, p. 425 , note 1) n'aurait-il pas pu imaginer une accélération si forte :

« [è] indubbio che nell'italiano postunitario si siano avute notevoli trasformazioni nella sintassi del verbo: per quanto riguarda i modi del verbo si assiste ad un processo di espansione dell'indicativo a spese del congiuntivo e del condizionale.»
[...] il est incontestable que dans l'histoire de la langue, après l'unité de l'Italie, il s'est produit d'importantes transformations dans la syntaxe du verbe. En ce qui concerne les modes, on observe une extension de l'indicatif au détriment du subjonctif et du conditionnel.

\section{Modalité épistémique}

La modalité que nous nous proposons d'examiner est celle qui indique les croyances et le savoir de l'énonciateur (cf. Nølke 2003). Nous nous bornons à cette aire sémantique dans la mesure où nous sélectionnons les verbes épistémiques conçus comme des assertifs modalisateurs. En français : croire, penser, imaginer, avoir l'impression... En italien : credere, pensare, immaginare/immaginarsi, avere l'impressione et même sembrare, parere.

Ces verbes sont considérés comme des « recteurs faibles » (BlancheBenveniste 1982, apud Korzen 2003, p. 116), à savoir de simples indicateurs de subjectivité « dont la fonction rappelle celle de certains adverbes de phrase » (Korzen 2003, p. 116).

En italien, Bronzi (1977, p. 432) utilise la dénomination " verbi assertivi deboli » (« di giudizio problematico »). Schneider (1999, p. 76) emploie le terme «modalizzanti deboli » et explique le rôle synonymique de ceux-ci :

[c] on un modalizzante debole il parlante esprime una riserva nei riguardi della verità della dipendente. La riserva è generica: non include informazione né sul suo grado né sul suo motivo e ancora meno sull'importanza soggettiva dello stato di cose descritto. Dato il suo carattere neutro e generico, un modalizzante debole può essere sostituito da un altro modalizzante debole, senza che ciò provochi un sostanziale mutamento del significato dell'enunciato. I seguenti verbi reggenti esprimono tutti press'a poco la stessa riserva: credo/mi pare/penso/mi sembra che Emma abbia l'otite.
[...] au moyen d'une légère modalisation, le locuteur exprime une réserve quant à la vérité de l'idée regardée qui en dépend. La réserve est d'ordre général, elle n'implique aucune information sur le degré, la motivation, et encore moins sur l'importance subjective de ce dont il est parlé. Étant donné sa neutralité et sa généralité, une faible modalisation peut être remplacée par une autre modalisation de même type, et cela sans provoquer un changement notable dans le signifié de l'énoncé. Les verbes recteurs suivants expriment tous plus ou moins la même réserve : credo/mi pare/penso/mi sembra che Emma abbia l'otite ("je crois/j'ai l'impression/ je pense / il me semble que Emma a*aie une otite)] 
Le caractère « désamorcé » d'un modalisateur faible permettrait donc la substitution avec un autre modalisateur du même type.

\section{Solutions sémantique et stylistique}

\subsection{Facteur réalité et facteur style en français}

L'usage a développé une distinction entre les cas où le choix de l'alternance indicatif $\left(\mathrm{IND}_{\mathrm{f}}\right.$ ) et subjonctif (SUBJ) est d'ordre sémantique et les cas où ce n'est que le style qui l'emporte.

Dans le cas du français, qui va être examiné de manière moins approfondie que l'italien en raison de l'étendue du phénomène, il existe un facteur réalité (Börjeson 1966, p. 5) et un facteur style (Foulet, apud ibid., p. 6) : «... dans la majorité des cas, semble-t-il, il n'est pas question d'une différence de sens, même ténue : il ne s'agit que d'un simple procédé de style ».

La participation du locuteur au contenu de l'énoncé peut jouer un rôle important. Pierre ne croit pas que Jean est venu serait aussi adéquat que Pierre ne croit pas que Jean soit venu. "Dans le premier cas on se trouve devant un fait : je sais bien, et mon interlocuteur sait également, que Jean est venu. Pourtant, Pierre ne le croit pas. Tandis que, dans le second cas, on partage l'incertitude de Pierre ». (Börjeson 1966, p. 25)

L'alternance est liée à une différence de portée de la négation (Kampers-Mahne 2003, p. 82-83) : avec le SUBJ la négation porte sur toute la phrase, le sujet ne considérant pas la subordonnée comme vraie (cf. possibilité de reprise de la phrase au SUBJ : Pierre ne croit pas que Jean soit venu $\rightarrow$ Pierre ne croit pas; de la phrase à l'IND : Pierre ne croit pas que Jean est venu $\rightarrow$ Pierre ne le croit pas).

À la solution en termes de polyphonie externe ( $\left(\mathrm{IND}_{\mathrm{f}}\right)$ et interne (SUBJ) (cf. Nølke 1993), nous préférons la théorie de Börjeson, qui exploite la distinction réalité/style.

Ce francisant (p. 26) soulève le problème du facteur opposition, qui expliquerait certains usages contraires à la norme, que nous énonçons cidessous :

- A. [phrase matrice exprimant une idée de jugement critique ${ }^{3}$ assertif

3. Curat (1991, p. 116) a recours à l'idée regardante de connaissance. Nous optons dans le présent article pour la notion de jugement critique qui englobe, à nos yeux de manière plus adéquate, les verbes paraître et sembler. Dans Soliman (2002), dans le compte rendu d'une expérience didactique portant sur le SUBJ qui visait à simplifier toutes les astuces et les ficelles fournies généralement aux étudiants italophones pour employer le bon mode, la terminologie dont Curat se sert est respectée. 
(positif) suivie d'une sous-phrase exprimant une idée actuelle (INDf)]

- B. [phrase matrice exprimant une idée de jugement critique antiassertifiant (négatif/interrogatif) suivie d'une sous-phrase exprimant une idée virtualisée ${ }^{4}$ (SUBJ)].

Sur ce scénario normatif, où [A] n'est pas susceptible de modifications, l'idée positive de la seconde partie des énoncés [1] et [2] contrôlerait, selon le linguiste suédois, "à rebours », par une sorte d'anticipation, l'idée négative initiale (modalité anti-assertifiante) :

[1] Mais je ne crois pas que l'indifférence actuelle persistera longtemps (apud Börjeson 1966, p. 9). ${ }^{5}$

[2] Certes, je ne crois pas qu'elle vous a conseillé de trahir votre pays. En revanche, elle eût dû se rendre compte que son goût du luxe vous obligeait à des dépenses inconsidérées (apud Börjeson 1966, p. 26).

Considération créative, mais contraire au rôle généralement admis du mode, qui est un problème de visée (cf. Guillaume 1929).

L'emploi de l'indicatif après une idée de jugement critique négatif est un trait de l'oralité qui n'aurait pas besoin d'une justification fouillée. D'après ce même linguiste (1966, p. 27), dans la langue parlée, je ne crois pas, je ne pense pas sont souvent utilisées comme des expressions toutes faites dans le but de moduler la négation non: Non, je n'pense pas; non, je crois pas. Ce serait plus vraisemblablement ce caractère d'expression cristallisée qui légitime je ne crois pas, je ne pense pas suivis d'une sousphrase à l'IND . .

L'emploi de l'IND ${ }_{\mathrm{f}}$ après une idée de jugement critique interrogatif (modalité anti-assertifiante) est possible dans l'interrogation avec inversion (pas de SUBJ après est-ce que, rarement après l'ordre progressif6). Dans les exemples [3] et [4], l'énonciateur s'interroge uniquement sur l'opinion de son co-énonciateur, alors que l'usage du SUBJ aurait comporté une extension de l'interrogation sur le contenu de la sous-phrase (Kampers-Mahne 2003, p. 85).

[3] Mais croit-on que la surveillance maritime française s'en tient aux limites des eaux territoriales ? (apud Börjeson 1966, p. 35)

[4] [...] ne pensez-vous pas que cette plaisanterie a assez duré? (apud Börjeson 1966, p. 36).

4. La quiddité du SUBJ est pour Curat (1991, p. 114) et Lagerqvist (2003, p. 153) sa capacité de virtualiser l'action.

5. Les énoncés que Börjeson fournit sont tirés de la presse.

6. L'interrogation avec est-ce que est une demande orientée, tandis que l'interrogation totale intonative est une demande de confirmation. (cf. Abouda 2002) 
Par conséquent, nous nous alignons sur Lagerqvist (2003, p. 159) qui témoigne de ce qu'il définit comme "symétrie modale ", à savoir une banalisation de l'effet de sens du choix modal employé :

[5] Pensez-vous qu'une loi suffira à éradiquer... le dopage? (apud Lagervist 2003, p. 159)

[6] Pensez-vous que cette Coupe d'Europe se résumera à un duel francoanglais? (ibidem)

Les exemples [3] à [6] et en particulier [7] révéleraient un emploi de l'INDi qui implique une orientation polarisée de la réponse du coénonciateur (avec ou sans droit de parole) :

[7] « Pensez-vous vraiment que j'ai réuni tout ce matériel dans le simple but de me mêler d'une guerre qui ne me concerne pas, qui ne m'a jamais concerné? Non. Le brouillage est là pour attirer quelqu'un. Vous, en l'occurrence. C'est toujours vous qui venez, j'ignore pourquoi » (Dunyach 2007, p. 99).7

\subsection{Facteur réalité et facteur style en italien}

Puisque l'emploi de l'IND $D_{i}$ au lieu du CONG semble être généralement attribué à l'oralité, il nous a paru intéressant de vérifier les retombées de cet usage sur l'écrit. Pour ce faire, l'exploration d'un corpus s'est avérée nécessaire. Notre choix s'est porté sur un corpus d'ordre littéraire : du genre pulp (les ouvrages de Niccolò Ammaniti ${ }^{8}$, qui s'aventure dans les zones les plus sombres de la condition humaine) au genre du « roman générationnel » (les ouvrages de Enrico Brizzi ${ }^{9}$ et de Giuseppe Culicchia ${ }^{10}$, qui livrent une radioscopie intégrale de la génération moderne et de ses faiblesses). Les histoires, qui décrivent une réalité existentielle inquiète, gravitent autour de clivages sociaux et de conflits générationnels. La parole est donc au peuple dans toute son authenticité11.

Les facteurs sociaux, donc les caractéristiques sociologiques des locuteurs, entrent en jeu, mais l'hypothèse que nous établissons est que le problème concerne la variation liée à la situation de communication.

Le chef de classement de notre corpus sera donc l'interaction/noninteraction, afin de vérifier l'emploi effectif du non-congiuntivo (NONCONG) dans les dialogues.

7. Dunyach, J.-C. (2007), Séparations, Nantes, L'Atalante.

8. Ammaniti, N. (Rome, 1966 -).

9. Brizzi, E. (Bologne, 1974 -).

10. Culicchia, G. (Turin, 1965 -).

11. Comme l'a déclaré René de Ceccatty, critique du Monde, cette littérature s'ouvre à la langue et aux jargons des classes sociales moyennes-basses (Di Stefano, P., «Italiani. Una critica scritta in francese», Il Corriere della sera, 27 janvier 2002). 
Résumons la norme préconisée en italien :

[phrase matrice exprimant une idée de jugement critique assertif/nonassertifiant suivie d'une sous-phrase exprimant une idée virtualisée (CONG)]

Si le facteur réalité est de mise, la sélection modale est effectuée par le locuteur qui suit sa propre logique : il évalue la validité de l'énoncé ou la possibilité d'existence de la circonstance, du fait décrits. L'emploi du NON-CONG n'est donc pas un faux pas :

[7a] Penso che sei triste. («tu as l'air triste» ou «je me rends compte que, avec tout ce qui a dî se passer, tu ne peux ne pas être triste »)

[7b] Penso che tu sia triste. («j'ai l'impression que tu as des raisons valables pour être triste») (cf. Sensini 1997, p. 268)

D'après Brugger et D'Angelo (1995, p. 199), l'INDi est le mode de la vérité, alors que le CONG est le mode du non-engagement: « [...] [w]ith the subjunctive the speaker does not take any attitude towards the content of the complement clause ».

L'idée de fausseté n'est pas exclue : dans [8], par exemple, l'effet de la drogue décrit à l'aide du CONG implique la fausseté du jugement de l'expérience vécue:

[8] La chiamano Spiderman perché uno dei tanti effetti che ti fa è che ti sembra di camminare sui muri, un altro è che, se dopo averla presa ti portano all'anagrafe e ti mettono in una fila che non finisce mai e ti dicono: "Vai a ritirare il certificato di nascita di Carleo » e tu non hai la più squallida idea di chi sia, lo fai, felice come una pasqua, e quando ci ripensi negli anni a venire continui a credere che quella sia stata l'esperienza più divertente della tua vita. (Ammaniti, Ti prendo e ti porto via, 1999, p. 300)

On l'appelle Spiderman car l'un de ses nombreux effets est qu'on a l'impression de grimper aux murs, et si on vous emmène à l'état civil et qu'on vous place dans une queue qui n'en finit pas en vous disant "Va retirer l'extrait de naissance de Carleo » sans que vous ayez la moindre idée de qui il peut bien s'agir, vous le faites, heureux comme un roi, et en y repensant les années suivantes, vous continuez à croire que ce fut l'expérience la plus amusante de toute votre vie.

En revanche, examinons les échantillons assertifs [9] et [10] :

[9] Neanche questo, Oscar, sapeva, e in ogni caso l'angoscia gli suggeriva di credere che Martina aveva impiegato il silenzio come una specie di copertura per i suoi incontri - qualunque fosse lo statuto di quelle occasioni - con un uomo che, nella sua vita, con ogni evidenza doveva aver contato, fin lì, almeno, ben più di lui. (Brizzi, Elogio di Oscar Firmian e del suo impeccabile stile, 1999, p. 277)

Cela non plus, Oscar ne le savait, et de toute manière l'angoisse lui suggérait de croire que Martine avait employé le silence comme une sorte de couverture pour ses rencontres - quel que fût le statut de ces occasions - avec un homme qui, dans sa vie, devait avoir compté, du moins jusque là, bien plus que lui.

[10] Rimangono in silenzio, seduti sul muretto, e se da una parte Pietro pensa che la sua amica è più bella del solito con quella maglietta di spugna 
azzurra, dall'altra sente il panico crescere.(Ammaniti, Ti prendo e ti porto via, 1999, p. 10)

Ils restent en silence, assis sur le muret et si, d'une part, Pierre trouve que son amie est plus belle que jamais dans son tee-shirt en éponge bleu clair, de l'autre, il sent monter en lui la panique.

Le facteur réalité n'entre point en jeu. Les énoncés [9] et [10] sont des cas de « décomposition » (Schmitt Jensen 1970, p. 244; vs. «cohésion»), où un simple soulignement de l'indépendance de la complétive à l'INDi se produit.

Prenons les non-assertifiants:

[11] Erica gli si addormenta nuda tra le braccia. Graziano, immobile per non svegliarla, la stringe e non può credere che quella ragazza così bella sia la sua donna. (Ammaniti, Ti prendo e ti porto via, 1999, p. 43).

Erica s'endort nue dans ses bras. Graziano, immobile pour ne pas la réveiller, la serre et ne peut croire que cette si belle fille soit sa femme.

[12] Miele non ci credeva, come poteva credere che quell'idiota gli stesse puntando la Beretta contro? (Ammaniti, Ti prendo e ti porto via, 1999, p. 180).

Miele n'en revenait pas, il ne pouvait pas croire que cet idiot était en train de pointer son Beretta sur lui.

Dans [11] et [12] les faits des complétives sont réels, mais le CONG valorise la distance du locuteur vis-à-vis de l'événement qui apparaît comme difficile à concevoir.

[13] «[...] Va be', Chiara, non ci ho mai creduto che eri felice con Ambris, e se tornavi al Man's Ruin o al Carthago era giusto per ricordare al mondo che ancora eri in giro. Di' di no » (Brizzi, Tre ragazzi immaginari, 1998, p. 115)

Le non-assertifiant non crederci [13] est aussi fort que la répétition du verbe dans [12]. Néanmoins, le mode change. L'univers de l'interaction y

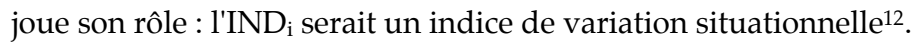

L'usage flottant entre $\mathrm{IND}_{\mathrm{i}}$ et CONG peut découler : i) du rôle plat des verbes épistémiques : comme le déclare Wandruszka (1991, p. 434), « la funzione comunicativa della subordinata si avvicina a quella di un'asserzione solo leggermente indebolita » [la fonction communicative de la subordonnée se rapproche d'une assertion à peine affaiblie]. C'est le contenu de la sous-phrase qui devient essentiel et qui admet la possibilité d'une valeur parenthétique du verbe régissant; ii) de la simple capitulation du CONG que justifierait la "symétrie modale » (cf. Lagerqvist 2003, p. 160) : " c'est une substitution qui implique l'abandon de la mise en pespective ». L'effet direct est la neutralisation sémantique du choix entre $\operatorname{IND}_{i}$ et CONG. Voici ce qu'en pense Prandi (2002, p. 38) :

12. À savoir la variation stylistique de Labov. 
[i]n caso di alternanza tra indicativo e congiuntivo in presenza di una stessa accezione di uno stesso verbo, cambia il registro stilistico dell'espressione, ma non cambia il valore modale della completiva, dato che non cambiano né il contenuto del verbo principale né la relazione grammaticale, che sono gli unici fattori pertinenti.

\begin{abstract}
dans le cas d'une alternance entre indicatif et subjonctif avec le même verbe et dans le même contexte, le registre stylistique est modifié, mais la valeur modale de la complétive ne l'est pas, étant donné que ni le contenu du verbe de la principale ni la relation grammaticale n'ont changé, et ce sont là les seuls facteurs pertinents.
\end{abstract}

En examinant les échantillons qui font l'intérêt de notre étude, nous signalons le contexte interactionnel des énoncés avec credere en modalité assertive [14] et anti-assertifiante [15]:

[14] Ha preso un disco e lo ha messo sul giradischi. "Così credono che suono.» (Ammaniti, Io non ho paura, 2001, p. 136)

Il a pris un disque et l'a placé sur le tourne-disque. "Comme ça ils croient que je joue».

[15] «Credevi che era facile ? Ma per fare queste cose bisogna avere sotto dei coglioni grossi come palloni da basket. E tu non ce l'hai »(Ammaniti, Fango,1996, p. 256)

"Tu pensais que ça serait facile? Mais pour faire ces choses-là il faut avoir des couilles comme des ballons de basket. Et tu n'en as pas".

Avec pensare en modalité assertive ([16 - 18]) et hypothétique ([19]) :

[16] « [...] Prima credevo di essere pazzo. Poi ho pensato che c'era il fantasma Formaggino. E invece eri tu » (Ammaniti, Io non ho paura, 2001, p. 149)

«[...] Au début, j'ai cru que j'étais fou. Puis j'ai pensé que c'était le fantôme Formaggino. Et enfin j'ai découvert que c'était toi »

[17] [...] «A te non ti è piaciuto il regalo?» «Sì. Ma pensavo che era un'altra cosa » (Ammaniti, Io non ho paura, 2001, p. 169)

[...] «Tu n'as pas aimé le cadeau? » «Oui. Mais je croyais que c'était autre chose ».

[18] «Perché ci hai messo tanto? Pensavo che eri tornato giù » (Ammaniti, Io non ho paura, 2001, p. 21)

«Pourquoi as-tu mis si longtemps? J'ai cru que tu étais redescendu».

[19] «D'accordo» mormori senza convinzione. «Se pensi che me la cago e non vuoi neppure fare a cazzotti, non ci parlo più con te " (Brizzi, Nessuno lo saprà, 2005, p. 104)

"D'accord", tu murmures sans conviction. "Si tu ne veux pas te battre parce que tu penses que j'ai peur, je ne t'adresse plus la parole »

Une fonction modalisatrice spéciale du verbe épistémique est détectable à la première personne:

[20] «Sono d'accordo con lei, credo che oramai c'è poco da fare...» dissi e poi cercando di dare un tono rilassato e oggettivo alle mie parole, continuai: 
«Anche chi è dotato di una volontà di ferro deve, in ogni caso, fare i conti con una struttura marcia e lottizzata e adeguarsi. [...]» (Ammaniti, Fango, 1996, p. 180).

[...] "Je suis d'accord avec vous. Je crois qu'il n'y a plus grand-chose à faire... » dis-je, puis, cherchant à donner un ton détendu et objectif à mes propres mots, je poursuivis : "même celui qui est doté d'une volonté inébranlable doit, de toute manière, se confronter à une structure pourrie et parcellisée et s'adapter [...]».

[21] «Come ti senti?»

«Male. Molto male. Credo che questa è stata la botta finale per la mia salute malridotta » (Ammaniti, Branchie, 1997/2006, p. 106)

«Comment te sens-tu?

"Mal. Très mal. À mon avis, cette fois, c'est le coup de grâce porté à ma pauvre santé ».

$[22] \ll[\ldots]$ Ho provato a bussare ma non ha risposto nessuno. Io credo che la vecchia ha stirato dentro l'appartamento. Dovete andare a vedere » (Ammaniti, Fango, 1996, p. 264)

" J'ai essayé de frapper à la porte, mais personne n'a répondu. Je pense que la vieille a clamsé dans l'appartement. Vous devez aller voir».

La valeur de credere est semblable à celle d'un adverbe signalant l'opinion personnelle ou le jugement subjectif : secondo me, per me. Alternativement, io credo équivaudrait à une nouvelle assertion du type «è quel che credo» portant sur la phrase qui précède (ex-complétive). Le verbe épistémique remplit dans les deux cas la fonction d'un modalisateur du discours de $\operatorname{soi}^{13}$.

Les autres personnes, elles, activent une fonction qui s'apparente à celle des verba dicendi introduisant des discours rapportés. Le cas de la deuxième personne est emblématique:

[24] Pensi che oltre la montagna, simile a una ferita verde fra le alture, deve stendersi la piana irrigata della Val di Chiana. (Brizzi, Nessuno lo saprà, 2005, p. 140)

[...] «Considérez que, par-delà la montagne, telle une blessure verte au milieu des collines, doit s'étendre la plaine irriguée de la Val di Chiana».

[25] Pensi che il Vietnamita vuole fare il misterioso, e pensi che i cerotti Secundapelle resistono alla doccia. Dovrebbero rilasciare un imprecisato principio attivo e, nel giro di pochi giorni, fondersi con il corpo, ma tu con calma prendi a lavorarne i margini »(Brizzi, Nessuno lo saprà, 2005, p. 191)

[...] « Considérez que le Vietnamien veut faire le mystérieux, et que les sparadraps Secundapelle résistent à la douche. Ils sont censés relâcher un principe actif non précisé, et se fondre avec le corps en l'espace de quelques jours, mais tu commences déjà calmement à en travailler les bords ».

13. Sauf les cas de discours direct libre à la première personne (« je me suis dit ») :

[23] Alla prima mi ero rivolto perché pensavo che sarei andato a vivere a Parigi. Alla seconda perché mi piacevano gli spot pubblicitari del Martini e pensavo chissà, magari riesco a inserirmi nel ramo promozione e propaganda (Culicchia, Tutti giù per terra, 1994, p. 113) 
Outre credere et pensare, nous avons effectué le dépouillement du verbe immaginarsi :

[26] Una volta il Triglia, in un improvviso attacco di loquacità, gli aveva spiegato: «Vedi, è semplice. Immaginati che c'è una che ti vuoi fare a una festa, quella chiaramente sta bevendo il suo gin-tonic o un'altra bibita alcolica, tu ti piazzi vicino e appena quella lascia incustodito il bicchiere o si volta le butti dentro una pasticca che dico io e il gioco è fatto. [...]» (Ammaniti, Ti prendo e ti porto via, 1999, p. 299)

[Un jour, dans un soudain accès de loquacité, le Rouget lui avait expliqué : "Tu vois, c'est simple. Imagine qu'il y a une femme que tu veux te faire. Vous êtes à une fête, elle est en train de boire son gin tonic ou une autre boisson alcoolisée; tu te places près d'elle et dès qu'elle laisse son verre sans surveillance ou qu'elle se tourne, tu jettes dedans un cachet à moi et les jeux sont faits. [...]»

Le verbe immaginarsi $+\mathrm{IND}_{\mathrm{i}}$ est également présent dans des contextes non-interactionnels :

[27] Mi immaginavo che gli zingari erano una specie di nanetti velocissimi, con le orecchie di volpe e le zampe di gallina. E invece erano persone normali. (Ammaniti, Io non ho paura, 2001, p. 92)

Je croyais que les tziganes étaient une espèce de petits nains très rapides, avec des oreilles de renard et des pattes de poule. Et bien non, c'étaient des personnes normales.

[28] A quel punto m'immaginavo che la mia pancia si apriva, un lungo taglio si spalancava e loro ci entravano dentro tutti tranquilli. (Ammaniti, Io non ho paura, 2001, p. 119)

À ce moment-là, j'imaginais que mon ventre s'ouvrait, une longue entaille se formait et eux y entraient tranquillement.

Les structures sembra/mi sembra ${ }^{14}$ (en modalité assertive et non) dans des contextes interactionnels régissent l'IND $\mathrm{IN}_{\mathrm{i}}$ :

[29] « Che è successo? Sembra che hai fatto il bagno in una piscina con tutti i panni addosso. Copriti. Stai battendo i denti. [...]» (Ammaniti, Fango, 1996, p. 170)

"Que s'est-il passé? On dirait que tu t'es baigné dans une piscine entièrement habillé. Couvre-toi. Tu claques des dents [...]»

[30] «Bene, mi sembra che sugli ctenofori è preparato. Può smettere » disse Ermini. (Ammaniti, Fango, 1996, p. 203)

«Bon, on dirait que vous êtes préparé sur les Cténophores. Vous pouvez vous arrêter » dit Ermine.

14. Le changement de mode que les structures équivalentes il semble $+\mathrm{SUBJ} / \mathrm{il}$ me semble + INDf comporte en français est absent en italien où l'on s'attend toujours au CONG. Avec il semble que (« les apparences donnent à penser que») sont exprimés le doute, la possibilité de faux jugement. Par contre, avec il me semble que ("j'ai l'impression que ») l'énonciateur prend en charge ses propos. Quant à il paraît que (« le bruit court que, selon ce qu'on dit»), il me paraît que («j'ai l'impression que »), ces tournures régissent l'INDf, car c'est la vérité, publique ou privée, qui est déclarée. 
[31] «Non mi sembra che stai tanto male, tutto sembra rispondere » (Ammaniti, Branchie, 1997/ 2006, p. 55)

["Je n'ai pas l'impression que tu vas si mal que ça, tout semble aller pour le mieux»]

Si le contexte interactionnel présente généralement le mode $\mathrm{IND}_{\mathrm{i}}$, le contexte non-interactionnel est émaillé d'occurrences alternées à l'IND ${ }_{\mathrm{i}}$ [32-35] et au CONG [36-39] :

[32] A cena nessuno parlava. Sembrava che ci stava il morto in casa. (Ammaniti, Io non ho paura, 2001, p. 58)

Au dîner, personne ne parlait. On aurait dit qu'il y avait un mort à la maison.

[33] Eravamo alti uguale e sembrava che avevamo la stessa età. (Ammaniti, Io non ho paura, 2001, p. 72)

Nous avions la même taille et nous semblions avoir le même âge.

[34] Di lì a poco non riuscivo a vedere niente, ma sembrava che la musica veniva dalla casa.(Ammaniti, Io non ho paura, 2001, p. 105)

Peu après, je ne pouvais plus rien voir, mais il semblait que la musique venait de la maison.

[35] Sembrava più vecchio, tra i capelli neri ne spuntava qualcuno bianco, le guance gli si erano scavate e sembrava che non dormiva da una settimana. (Ammaniti, Io non ho paura, 2001, p. 110)

[36] È rimasta in silenzio. Sembrava che non se ne volesse più andare. Si è girata e ha sussurrato un grazie. (Ammaniti, Io non ho paura, 2001, p. 103)

Elle est restée en silence. On aurait dit qu'elle ne voulait plus partir. Elle s'est retournée et a murmuré un merci.

[37] Sembrava che stesse soffocando. (Ammaniti, Io non ho paura, 2001, p. 115)

[38] Sembrava che se ne volesse andare, poi si è riseduto sul letto. (Ammaniti, Io non ho paura, 2001, p. 161)

On aurait dit qu'il voulait s'en aller, puis il s'est rassis sur le lit]

[39] Sembrava che il cielo si fosse bucato. Nel fragore dei tuoni, i lampi univano le nuvole con la terra. (Ammaniti, Io non ho paura, 2001, p. 184)

Un cas isolé de parere suivi de $\mathrm{l}^{\prime} \mathrm{IND}_{\mathrm{i}}$ [40] ne serait pas, à en croire Schneider (1999, p. 97), sémantiquement différent de parere suivi du CONG. La décomposition serait en action : parere $+\mathrm{IND}_{\mathrm{i}}$ constituerait tout simplement une structure à mi-chemin entre une sous-phrase au CONG et une phrase avec mi pare parenthétique. Nous risquons l'hypothèse cognitive d'une image mentale qui se manifeste à l'esprit de manière plus vive :

[40] Anche quando mi aprivo un varco nei resti di quel mare di spighe destinato a essere stipato nelle balle e intorno non avevo che cielo, mi pareva che mille occhi mi guardavano. (Ammaniti, Io non ho paura, 2001, p. 173)

[Même quand je me frayais un passage dans les restes de cette mer d'épis destinés à être enserrés dans des ballots et qu'autour de moi je n'avais que le ciel, il me semblait que des milliers d'yeux me regardaient]. 


\subsection{Modulation de l'opération traduisante}

La fréquente platitude sémantique du choix modal à laquelle nous avons fait allusion peut être remarquée dans la pénurie d'effets compensatoires que les traducteurs-réécrivains créent dans les traductions publiées des ouvrages analysés. En effet, dans la plupart des cas, ils ne signalent dans le texte d'arrivée l'écart de la norme, difficile à définir, ni au niveau nucléaire, c'est-à-dire syntaxique - ce qui est plausible en raison de la syntaxe du français (cf. §3.1, [A]) - ni au niveau périphérique, à savoir dans le trans-verbe. Pour ne citer que quelques exemples:

[41a] « Li trovo un po' macabri... ma nonostante questo credo che hai una pennellata originale. Vai avanti...» (Ammaniti, Fango, 1996, p. 148)

" Je les trouve un peu macabres... mais je crois pourtant que tu as un coup de pinceau original. Il faut que tu continues... »

[42a] I corridori perdono perché credono che la vittoria ha un senso. La vittoria non ha senso. L'obiettivo non è la vittoria. L'obiettivo è pedalare. Fausto Coppi gli stava parlando. Pedalare fino a schiattare. (Ammaniti, Ti prendo e ti porto via, 1999, p. 339)

Les coureurs perdent parce qu'ils croient que la victoire a un sens. La victoire n'a pas de sens. L'objectif n'est pas la victoire. L'objectif, c'est de pédaler. Fausto Coppi lui parlait. Pédaler à en crever. (Ammaniti, Et je t'emmène, 2001, p. 381)

[43a (21)] «Come ti senti? »

"Male. Molto male. Credo che questa è stata la botta finale per la mia salute malridotta ». (Ammaniti, Branchie, 1997/2006, p. 106)

"Comment te sens-tu? »

"Mal. Très mal. À mon avis, cette fois, c'est le coup de grâce porté à ma pauvre santé ». (Ammaniti, Branchies, 1999, p. 110).

L'usage non-standard sembrare $+\operatorname{IND}_{\mathrm{i}}[44 \mathrm{a}]$ est rendu sans faire la lumière sur le différentiel syntaxique :

[44a]A cena nessuno parlava.

Sembrava che ci stava il morto in casa. (Ammaniti, Io non ho paura, 2001, p. 58)

Au dîner, personne ne parlait.

On aurait dit qu'il y avait un mort à la maison. (Ammaniti, Je n'ai pas peur, 2002, p. 70)

Avec le même verbe à la même modalité énonciative, l'élision du pronom personnel [45b] peut être considérée comme un simple effet compensatoire périphérique qui rend compte de l'oralité, et puisque le NON-CONG est d'abord un trait de l'oral, ce choix diamésique contrebalancerait, quoique petitement, la perte de l'écart de l'italien standard :

[45a] « [...] Sembra che ci credi, quando discuti di cose come l'aborto ». (Culicchia, Il paese delle meraviglie, 2004, p. 112)

"[...] On dirait que t'y crois, quand tu parles de choses comme l'avortement». (Culicchia, Le pays des merveilles, 2006, p. 133) 
Cas unique : [46b], où le NON-CONG (après un verbe épistémique de la phrase matrice en modalité anti-assertifiante) est traduit au niveau nucléaire par un NON-SUBJ (cf. §3.1) et au niveau périphérique par une sorte d'ajustement général à la teneur du texte de départ (choix lexical informel) :

[46a] Gli mise le mani sulle spalle. « Li devi guardare negli occhi. E anche se hai una paura che te la fai sotto, non devi pensare che loro non ce l'hanno, sono solo più bravi di te a non mostrarla. [...] » (Ammaniti, Ti prendo e ti porto via, 1999, p. 364)

Il lui mit les mains sur les épaules. "Tu dois les regarder dans les yeux. Et même si t'as une trouille bleue à faire dans ton froc, crois pas qu'eux ils ont pas peur, c'est seulement qu'ils sont meilleurs que toi pour la cacher. [...]» (Ammaniti, Et je t'emmène, 2001, p. 409)

\section{Conclusions}

Le déclin du CONG en italien moyen (Sabatini 1985) n'est plus considéré comme un problème de langue non châtiée - Schmitt Jensen (1970, p. 180) dénonçait le phénomène comme un fait typique de «la langue parlée quelque peu négligée »-, mais bien comme une tendance générale affectant non seulement l'oral, mais aussi l'écrit. Généralement, le choix de l'IND $\mathrm{i}_{\mathrm{i}}$ figurant dans les complétives régies par des verbes épistémiques n'apparaît pas dans les échantillons linguistiques recueillis comme un choix de validation ou de non validation du contenu de la sous-phrase, mais plutôt comme une alternative stylistique qui reflète le caractère immédiat de la pensée. Ce choix, typique de l'interaction, délègue toute l'incertitude, que le CONG devrait véhiculer, au verbe recteur qui garde, lui seul, le sème de la réserve. Sans doute, nos quelques considérations en matière de traduction italien/ français corroborent-elles cette interprétation à cause de la pauvreté d'effets compensatoires, que le traducteurréécrivain compétent devrait ressentir le besoin d'opérer, du moins marginalement, en cas de choix non stylistique.

Università di Padova

Babel EA 2649

\section{Bibliographie}

\section{Ouvrages consultés}

ABOUDA, L. (2002), "Négation, interrogation et alternance indicatifsubjonctif », French Language Studies, 12, 1, p. 1-22.

BERRUTO, G. (1987), Sociolinguistica dell'italiano contemporaneo, Roma, Nuova Italia Scientifica.

BIRKELUND, M., Boysen, G. Søren Kjærsgaard, P. (eds) (2003), Aspects de la modalité, Tübingen, Niemeyer. 
BONOMI, I. (1993), " I giornali e l'italiano dell'uso medio », Studi di Grammatica Italiana, XV, p. 181-201.

BONOMI, I. (1996), «La narrativa e l'italiano dell'uso medio», Studi di Grammatica Italiana, XVIII, p. 321-338.

BORILLO, A. (2004), « Les 'Adverbes d'opinion forte' selon moi, à mes yeux, à mon avis,...: point de vue subjectif et effet d'atténuation », Langue Française, 142, p. 31-40.

BÖRJESON, L. (1966), « La fréquence du subjonctif dans les subordonnées complétives introduites par 'que' étudiée dans des textes français contemporains », Studia neophilologica, 38, p. 3-64.

BOYSEN, G. (2003), « Deux facteurs fondamentaux pour la syntaxe modale des complétives: personne grammaticale et fonction syntaxique », in Birkelund, M., Boysen, G., Søren Kjærsgaard, P. (eds) (2003), Aspects de la modalité, Tübingen, Niemeyer, p. 45-48.

BRONZI, A.M. (1977), «Indicativo e congiuntivo nelle completive italiane », Studi di Grammatica Italiana, VI, p. 425-450.

BRUGGER, G., D'Angelo, M. (1995), « Movement at LF triggered by mood and tense », Folia Linguistica, XXIX/3-4, p. 195-221.

CORTELAZZO, M.A. (2002), " La fisionomia dell'italiano oggi », in Schena, L., Soliman, L.T. (eds), Prospettive linguistiche della nuova Europa, Atti del Congresso Linguistico Internazionale Università Bocconi (Milano, 9-10 novembre 2001), Milano, Egea, p. 227-243.

CURAT, H. (1991), Morphologie verbale et référence temporelle en français moderne. Essai de sémantique grammaticale, Genève-Paris, Droz.

DARDANO, M., Trifone, P. (1995), Grammatica italiana con nozioni di linguistica, Bologna, Zanichelli.

Guillaume, G. (1929), Temps et verbe. Théorie des aspects, des modes et des temps, Paris, Champion.

KAMPERS-MANHE, B. (2003), « Le subjonctif dans les complétives: une mise au point », in Birkelund, M., Boysen, G., Søren Kjærsgaard, P. (eds) (2003), Aspects de la modalité, Tübingen, Niemeyer, p. 75-87.

KORZEN, H. (2003), " Subjonctif, indicatif et assertion ou: comment expliquer le mode dans les subordonnées complétives? ", in Birkelund, M., Boysen, G., Søren Kjærsgaard, P. (eds) (2003), Aspects de la modalité, Tübingen, Niemeyer, p. 113-129.

KRONNING, H. (2003), " Modalité et évidentialité », in Birkelund, M., Boysen, G., Søren Kjærsgaard, P. (eds) (2003), Aspects de la modalité, Tübingen, Niemeyer, p. 131-151.

LAGERQVIST, H. (2003), « L'autonomie du subjonctif français en proposition complétive ", in Birkelund, et al. (eds) (2003), Aspects de la modalité, Tübingen, Niemeyer, p. 153-166. 
LEPSCHY, A.L. \& LEPSCHY, G. (1977), The Italian Language Today, London, Hutchinson \& Co.

LEPSCHY, A.L. \& LEPSCHY, G. (1981), La lingua italiana, Milano, Bompiani.

MAILHAC, J.-P. (2000), « Sens, choix et subjonctif », French Language Studies, 10 , p. $229-244$.

MARTin, R. (1992, 2e éd.), Pour une logique du sens, Paris, PUF.

MAZZOLENI, M. (2002), « Il congiuntivo nel periodo ipotetico », in Schena, L., Prandi, M.,Mazzoleni, M. (eds) (2000), Intorno al congiuntivo, Bologna, Clueb, p. 65-81.

NØLKE, H. (1993), Le regard du locuteur, Paris, Kimé.

NØLKE, H. (2003), " Modalité(s) énonciative(s) adverbiale(s) », in Birkelund, M., Boysen, G.,Søren Kjærsgaard, P. (eds) (2003), Aspects de la modalité, Tübingen, Niemeyer, p. 181-192.

PATOTA, G. (2006), Grammatica di riferimento dell'italiano contemporaneo, Novara, De Agostani Scuola.

PRANDI, M. (2000), «C'è un valore per il congiuntivo? », in Schena, L. et al. (eds) (2000), Intorno al congiuntivo, Bologna, Clueb, p. 29-44.

QUAYLE, N. (ed) (1998), « Le subjonctif », Modèles linguistiques, XIX, 1.

ROCCI, A. (2007), «Epistemic modality and questions in dialogue. The case of Italian interrogative constructions in the subjunctive mood », in de Saussure, L., Moeschler, J., Puskas, G. (eds), Tense, Mood and Aspect. Theoretical and Descriptive Issues, Amsterdam-New York, Rodopi, p. 129-153.

SABATINI, F. (1985), « L"italiano dell'uso medio': una realtà tra le varietà linguistiche italiane », in G. Holtus, E. Radtke (eds), Gesprochenes Italienisch in Geschichte und Gegenwart, Tübingen, Narr, 154-184.

SAND, J.U. (2003), « Le subjonctif revisité », in Birkelund, M. - Boysen, G. Søren Kjærsgaard, P. (eds) (2003), Aspects de la modalité, Tübingen, Niemeyer, p. 193-201.

SCHENA, L., PRANDI, M.,MAZZOLENI, M. (eds) (2000), Intorno al congiuntivo, Bologna, Clueb.

SCHENA, L. (2000), «Per un approccio al congiuntivo francese in chiave psicomeccanica », in Schena, et al. (eds) (2000), Intorno al congiuntivo, Bologna, Clueb, p. 45-64.

SCHMITT JENSEN, J. (1970), Subjonctif et hypotaxe en italien, Odense, Odense University Press.

SCHNEIDER, S. (1999), Il congiuntivo tra modalità e subordinazione. Uno studio sull'italiano parlato, Roma, Carocci.

SENSINI, M. (1997), La grammatica della lingua italiana, Milano, Mondadori. 
SEVERGNINI, B. (2007), « Non calpestate i congiuntivi », in L'italiano. Lezioni semiserie, Milano, Rizzoli, p. 149-153.

SOLIMAN, L.T. (2000), « Modalità e implicazioni aspettuali : analisi contrastiva italiano/francese del congiuntivo nell'ottica psicomeccanica », in Schena, L.et al. (eds) (2000), Intorno al congiuntivo, Bologna, Clueb, p. 291-306.

SOUTET, O. (2000), Le subjonctif en français, Paris, Ophrys.

VENIER, F. (1991), La modalizzazione assertiva. Avverbi modali e verbi parentetici, Milano, Franco Angeli.

WANDRUSZKA, U. (1991), "Frasi subordinate al congiuntivo», cap. 8, in Renzi, L.,Salvi, G.,Cardinaletti, A. (eds), Grande Grammatica Italiana di consultazione. Vol. II: I sintagmi verbale, aggettivale, avverbiale. La subordinazione, Bologna, Il Mulino (nuova ed. 2001), p. 415-481.

\section{Corpus}

Ammaniti, N., Fango, Milano, Mondadori, 1996.

Ammaniti, N., Branchie, Torino, Einaudi, 1997/2006.

Ammaniti, N., Ti prendo e ti porto via, Milano, Mondadori, 1999.

Ammaniti, N., Io non ho paura, Torino, Einaudi, 2001.

Brizzi, E., Tre ragazzi immaginari, Milano, Baldini \& Castaldi, 1998.

Brizzi, E., Elogio di Oscar Firmian e del suo impeccabile stile, Milano, Baldini \& Castoldi, 1999.

Brizzi, E., Nessuno lo saprà. Viaggio a piedi dall'Argentario al Conero, Milano, Mondadori, 2005.

Culicchia, G., Tutti giù per terra, Milano, Garzanti, 1994.

Culicchia, G., Il paese delle meraviglie, Milano, Garzanti, 2004.

\section{Traductions}

Ammaniti, N., Dernier réveillon et autres nouvelles cannibales, traduit de l'italien par D. Vittoz, Paris, Hachette Littératures, 1998.

Ammaniti, N., Branchies ou l'épopée rocambolesque d'un héros prêt à tout malgré lui, traduit de l'italien par L. Berini, Paris, Editions du Félin, 1999.

Ammaniti, N., Et je t'emmène, traduit de l'italien par M. Bouzaher, Paris, Grasset \& Fasquelle, 2001.

Ammaniti, N., Je n'ai pas peur, traduit de l'italien par M. Bouzaher, Paris, Grasset \& Fasquelle, 2002.

Culicchia, G., Patatras, traduit de l'italien par F. Liffran, Paris, Payot \& Rivages, 1995.

Culicchia, G., Le pays des merveilles, traduit de l'italien par V. Raynaud, Paris, Albin Michel, 2006. 